\title{
STUDYING OF NICKEL ALLOYS WITH 1 at.\% OF Ge, Zn, In, Zr, AND Pb BY POSITRON ANNIHILATION METHODS
}

\author{
A. Baranowski ${ }^{a}$, M. Kostrzewa $^{b}$ and M. Szuszkiewicz ${ }^{c}$ \\ ${ }^{a}$ Institute of Experimental Physics, Wrocław University \\ Pl. Maxa Borna 9, 50-204 Wrocław, Poland \\ ${ }^{b}$ Physical Laboratory, Technical University of Opole \\ Ozimska 75, 45-370 Opole, Poland \\ ${ }^{c}$ Institute of Physics, University of Opole, Oleska 48, 45-052 Opole, Poland
}

The measurements of cold rolled nickel alloys with 1 at.\% of Ge, $\mathrm{Zn}$, In, $\mathrm{Zr}, \mathrm{Pb}$ and additionally $\mathrm{Ti}$ and $\mathrm{Sb}$ by positron lifetime and Doppler broadening techniques have been done. Monovacancies were a dominating type of defects in the alloys. The authors of the present paper connect the differences between the annihilation parameters of the investigated samples with the existence of the vacancy-impurity atom pairs. A direct proportionality between the vacancy lifetime and the product of vacancy-impurity binding energy and atomic radius of impurity atom has been found.

PACS numbers: 78.70.Bj, 78.30.Er

\section{Introduction}

Results of positron annihilation studies of dilute binary alloys (primary solid solutions) rarely show distinct differences with respect to those for pure metal (solvent). The measurable differences occur only when the presence of the admixture causes the changes in the defect or the electronic structure of solvent metal, the first of these two factors seems to play a dominating role. Several papers devoted to the positron annihilation studies of the vacancy-impurity $(\mathrm{v}-\mathrm{i})$ interaction in nickel have been already published yielding, among others, the detailed examination of the defect structure of alloys [1], calculations of the vacancy-impurity binding energy $\left(E_{\mathrm{vi}}^{\mathrm{B}}\right)$, e.g. [1-3], or an attempt to elaborate the vacancy-impurity interaction theory [4], though the last task was connected with some difficulties. Nevertheless, the subject remains to be not fully exhausted and every new attempt of approaching it may bring some new valuable information.

The main purpose of the present study was to investigate the v-i interaction in a series of nickel-based alloys (primary solid solutions) containing 1 at.\% 
of $\mathrm{Ge}, \mathrm{Zn}, \mathrm{In}, \mathrm{Zr}$ or $\mathrm{Pb}$ by two positron annihilation methods: positron lifetime and Doppler broadening measurements. Additionally, the potentialities of a newly constructed, modified apparatus for Doppler broadening measurements [5] were examined.

\section{Experimental}

The investigated alloys were produced by induction of $4 \mathrm{~N}$ purity nickel with 1 at.\% addition of pure $\mathrm{Zn}, \mathrm{Ge}, \mathrm{Zr}$, In, and $\mathrm{Pb}$. After melting the ingots were cut into flat, $2 \mathrm{~mm}$ thick pieces and submitted to long-lasting homogenisation tempering at $1000^{\circ} \mathrm{C}$ followed by slow cooling to the room temperature. Then the homogeneity and chemical composition of the alloys were checked by laser and electron beam microanalyses. The slices of the alloys were cold rolled in $0.1 \mathrm{~mm}$ steps to get finally $1 \mathrm{~mm}$ thick plates, from which the square samples with the area of $1 \mathrm{~cm}^{2}$ were cut.

A modernised version of spectrometer based on two HPGe detectors was used in Doppler broadening measurements to get differential spectra (the relation between the number of counts and energy difference of two registered annihilation photons). The differential spectra are analogous to those, which come from conventional, one-detector Doppler devices but they are symmetrical, a peak to background ratio is higher and the relative apparatus resolution can be better by the factor of $\sqrt{2}$. The differential spectrum as a one of the two-dimensional Doppler broadening result presentation was used, e.g. by Lynn et al. [6]. In our experimental setup, the peak to background ratio was about $5 \times 10^{4}$, and the relative energy resolution improved by the factor of 1.3 caused a decrease in the FWHM value to about $2 \mathrm{keV}$. As a source of positrons ${ }^{22} \mathrm{Na}$ was used. The average number of counts per spectrum was $4.8 \times 10^{6}$.

Positron annihilation lifetime measurements were performed at room temperature. The Ortec commercial system with $240 \mathrm{ps}$ time resolution was used. The positron source $\left({ }^{22} \mathrm{Na}\right)$ of the activity of $10 \mu \mathrm{Ci}$ was placed between two identical thin Hostaphan foils $\left(0.8 \mathrm{mg} \cdot \mathrm{cm}^{-2}\right.$ each) and then sandwiched between the investigated samples. The average amount of counts per spectrum was $1 \times 10^{7}$. The analysis of positron lifetime curves was performed using POSITRONFIT program [7] including source correction with contribution to be equal $4 \%$. In the second series, for all the samples mentioned above and additionally for NiTi and $\mathrm{NiSb}$, the lifetime measurements using another fast-slow coincidence device with ${ }^{22} \mathrm{Na}$ source of activity $2.5 \mu \mathrm{Ci}$ have been done. The time resolution of the device was determined as $300 \mathrm{ps}$ and the value of counts per spectrum was about $2 \times 10^{6}$.

\section{Results and discussion}

The investigations of the vacancies in metallic materials requires their presence in the amount exceeding the lower threshold for their detection by positron annihilation methods $\left(\approx 10^{-1} \mathrm{ppm}[8]\right)$, but in the studies of the v-i interaction the existence of detectable amount of vacancy-impurity atom pairs is important too. 
Severe cold rolling of the samples with $50 \%$ reduction in thickness guarantees not only the exceeding of the lower threshold of the sensitivity of annihilation technique but allows for expecting that the vacancy channel of annihilation will be dominant. In similar studies [9] of NiGe alloys the saturation of the annihilation in vacancies was achieved after the reduction of the sample thickness by $25 \%$. Therefore the observed differences in the annihilation parameters with respect to those for pure nickel indicate the presence of the $v-i$ pairs and contain information on the $\mathrm{v}-\mathrm{i}$ interaction in a given alloy.

Due to the very small differences between the annihilation spectra for investigated alloys, the presentation of the results of Doppler broadening measurements as ratio curves was chosen for the best visualisation of their dissimilarity (Fig. 1).

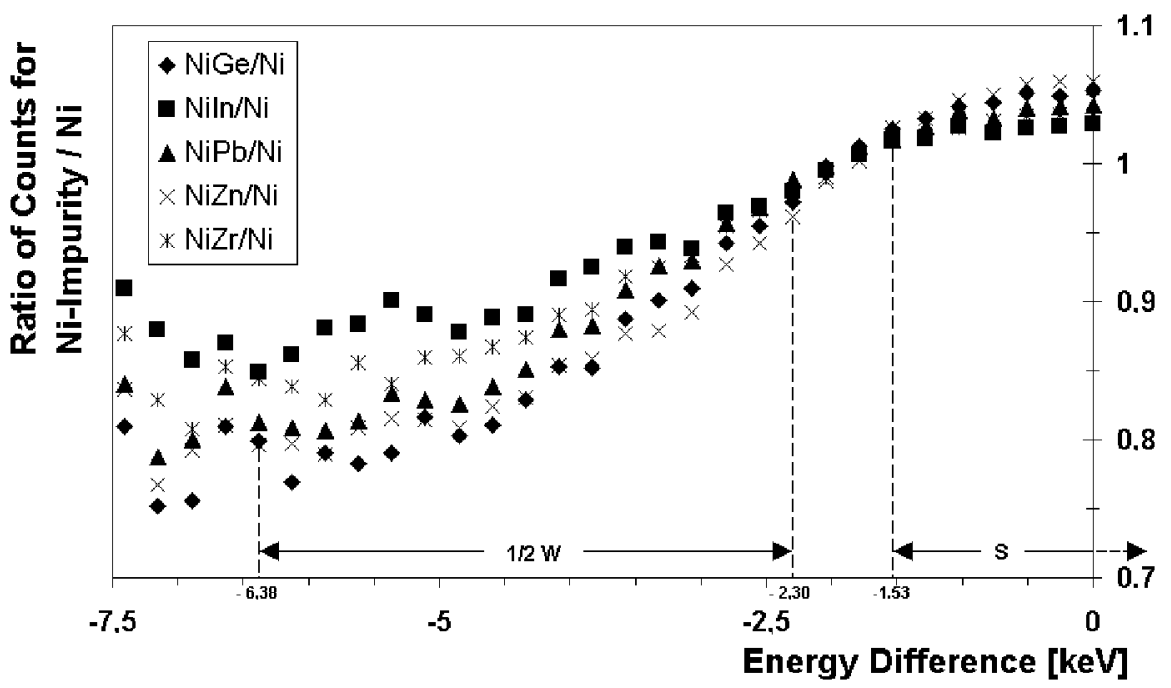

Fig. 1. The left sides of the ratio curves normalized to the equal number of counts for each of the spectra. The ranges of $W$ and $S$ parameters are also presented.

The ratio curves were obtained by normalisation of the raw spectra to the equal number of counts per spectrum followed by dividing the spectrum for a given alloy by the spectrum for the reference sample - pure, cold rolled nickel. As it follows from Fig. 1, in the whole range of the large energy differences, the location of data points for investigated alloys exhibits a remarkable regularity. The points representing the results for NiIn alloy are always located at the top and those for other alloys below, in the sequence NiZr, NiPb, NiGe, and NiZn. Therefore, it can be expected that the $W$ and $S$ parameters, averaging the measurement result presented in Fig. 1, reflect this characteristic situation. The results of the $W$ and $S$ parameters determination are presented in Table I.

As expected, the values of the $W$ parameter for investigated alloys decrease in the sequence NiIn, NiZr, NiPb, NiGe, NiZn. A strictly opposite trend in the values of $S$ parameter is observed. These regularities are clearly seen on the $S=f(W)$ diagram (Fig. 2) showing a linearity well known from other annihilation studies of 
TABLE I

The values of $S$ and $W$ parameters.

\begin{tabular}{c|c|c|c|c|c|c}
\hline \hline & $\mathrm{Ni}$ & $\mathrm{NiIn}$ & $\mathrm{NiZr}$ & $\mathrm{NiPb}$ & $\mathrm{NiGe}$ & $\mathrm{NiZn}$ \\
\hline$S$ & 0.5862 & 0.5990 & 0.6027 & 0.6057 & 0.6102 & 0.6127 \\
$W$ & 0.2816 & 0.2695 & 0.2663 & 0.2634 & 0.2588 & 0.2573
\end{tabular}

defects in solids. It should be noted that the commonly accepted explanation of the linearity of the $S=f(W)$ plot [10] concerns the sample in which concentration of only one type of defects is changed, e.g. by tempering, and the number of defects is kept within the limits of the sensitivity of positron annihilation techniques. For the investigated Ni-alloys these assumptions are not fulfilled, but the observed linearity of the $S=f(W)$ plot seems not to be fortuitous.

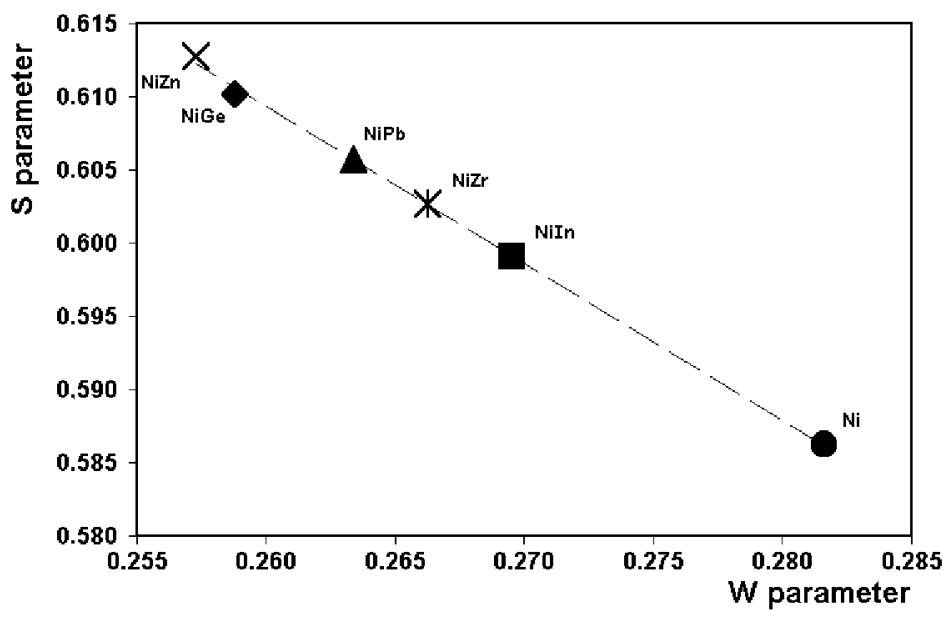

Fig. 2. $S=f(W)$ diagram for pure Ni and for the alloys.

Assuming that the increased value of $W$ parameter is connected with an increased contribution of positron annihilations with $d$ electrons of impurity atoms, we can interpret almost all of the presented results connecting them with the number of the outermost valence electrons shielding $d$ electrons of impurity atoms. But a high deviation from the rule for NiZn suggests that the kind of defects, likewise the number of defect-impurity pair, is of a great importance too. Apart from that, a low value of the $W$ parameter could not be caused by the lack of tendency of annihilations with deep bound electrons but by a high probability of annihilations with outermost, low-momentum electrons. In such situation the increase in $S$ parameter and this way decrease in $W$ parameter can be observed. It looks as if the recalling of lifetime results to lighten the question of a kind of defects would do an important step towards. 
The lifetime spectra (after correction for source contribution) were fitted with a sum of two exponentials. The first component was always dominant. In relation to the whole series of measurements the average intensity of the first component equals $85 \%$ and taking into account the range of lifetimes (163-184 ps) it seems that it can be regarded as the vacancy component. The average lifetime for the second component equals to $306 \mathrm{ps}$ and we think it corresponds to vacancy aggregates (clusters), grain boundaries or other defects formed during the procedure of the sample preparation. The intensity of this component amounts up to $15 \%$ in average. In fact the lifetime values of the second component range from $\sim 250$ ps to $\sim 400$ ps and the relative intensities range over almost $35 \%$. The constraint (306 ps) analysis is excluded due to chi-square behaviour (a too big value) and it states that the spectra are lacking in the possible remainder of the source component. Table II presents the results of lifetime measurements.

TABLE II

Average lifetime $\left(\tau_{\mathrm{av}}\right)$, first component lifetime $\left(\tau_{1}\right)$ and the intensity of the first component $\left(I_{1}\right)$ for investigated alloys.

\begin{tabular}{c|c|c|c|c|c|c|c|c}
\hline \hline & $\mathrm{Ni}$ & $\mathrm{NiGe}$ & $\mathrm{NiZn}$ & $\mathrm{NiTi}$ & $\mathrm{NiIn}$ & $\mathrm{NiSb}$ & $\mathrm{NiZr}$ & $\mathrm{NiPb}$ \\
\hline$\tau_{1}[\mathrm{ps}]$ & 163 & 168 & 171 & 172 & 179 & 180 & 181 & 184 \\
& $(1)^{*}$ & $(1)$ & $(4)$ & $(3)$ & $(4)$ & $(3)$ & $(4)$ & $(4)$ \\
\hline$I_{1}[\%]$ & 89.2 & 76.7 & 64.5 & 98 & 79.8 & 95 & 95.7 & 80.6 \\
& $(0.1)$ & $(0.3)$ & $(0.4)$ & $(0.5)$ & $(0.7)$ & $(0.8)$ & $(0.3)$ & $(0.3)$ \\
\hline$\tau_{\mathrm{av}}[\mathrm{ps}]$ & 177.5 & 198.3 & 204.7 & 174.6 & 196.2 & 185.2 & 191.1 & 209.8 \\
& $(1.2)$ & $(1.0)$ & $(3.9)$ & $(5.6)$ & $(6.3)$ & $(4.7)$ & $(6.7)$ & $(4.7)$ \\
\hline
\end{tabular}

*Standard deviations of measured quantities are given in round brackets.

It is worth noting that all the samples consist in $99 \%$ of nickel and the annihilation of positrons from defect state dominates (lack of the 108 ps component). Therefore if we observe small differences in measured lifetimes they should be connected with different (for each of the alloy) defects and with coexistence of defects and impurity atoms in the nearest surrounding. The high value of the first component lifetime in the case of $\mathrm{Pb}$ or $\mathrm{Zr}$ could mean that the majority of positrons annihilates in unbound vacancies, like in pure nickel but a small part of them annihilates from vacancies bounded with $\mathrm{Pb}$ or $\mathrm{Zr}$ atoms. Such Ni vacancy should has the size depending on the impurity atom attached. One can also assume an impurity atom bounded with vacancies existing in a bigger number with not necessarily much bigger dimensions. Considering that both the dimensions and the amount of the $\mathrm{v}-\mathrm{i}$ pairs affect the registered positron lifetime, and taking into account that the first lifetime component does not represent only the $\mathrm{v}-\mathrm{i}$ pairs because of a strong representation of the unbound vacancies (like in pure nickel), the evaluation of the effect of dimensions and population of the v-i pairs on the value of the first lifetime component seems to be impossible. 
Let us assume hypothetically that the first lifetime component data contain information only about dimensions of vacancies existing near impurity atoms. Then the correlation between the first lifetime component and the impurity atom radius would be expected. Impurity atoms deformed their surrounding, including vacancies, on the basis of lattice relaxation mechanisms. No such correlation has been found.

If the lifetime data contained only information about the number of $\mathrm{v}-\mathrm{i}$ pairs with only slightly different vacancy dimensions for all the samples then the correlation between the first lifetime component and the number of $\mathrm{v}-\mathrm{i}$ pairs would be expected. The establishing of such a correlation is more difficult because of the lack of direct information about the number of v-i pairs but with a certain probability it is possible to check the existence of such correlation by an indirect way. Namely there is a magnitude - the vacancy-impurity binding energy $E_{\mathrm{vi}}^{\mathrm{B}}$, which is, by some way, proportional to the number of v-i pairs. No correlation between the first lifetime component and $E_{\mathrm{vi}}^{\mathrm{B}}$ has been found too.

Therefore, the combination of dimension factor represented by the atomic radius of impurity atom with the v-i population factor represented by $E_{\mathrm{vi}}^{\mathrm{B}}$ with the relation to the first component lifetime suggests itself to be proven. The results of an attempt to find such a correlation is presented in Fig. 3.

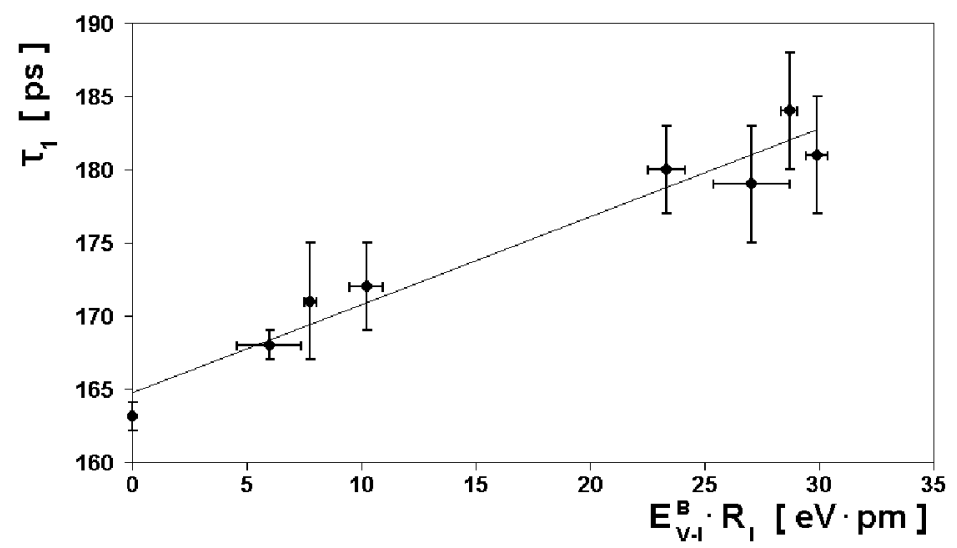

Fig. 3. First lifetime component versus product of the $E_{\mathrm{vi}}^{\mathrm{B}}$ and atomic radius of the impurity.

A direct proportionality between the $\tau_{1}$ and the $E_{\mathrm{vi}}^{\mathrm{B}} R_{i}$ product has been found. This way the assumption about influence of dimensions and population of v-i pairs on the annihilation characteristics seems to be confirmed.

The procedure leading to revealing the above correlation is not free of limitations. The assumption of proportionality between $E_{\mathrm{vi}}^{\mathrm{B}}$ and the number of v-i pairs in the sample requires some discussion. In fact, the method of $E_{\mathrm{vi}}^{\mathrm{B}}$ calculation (Górecki [1]), is based on the simple but useful models of the thermodynamics of vacancies in dilute binary alloys developed by Lomer [11] and Schapink [12]. These 
models describe the v-i interaction with the assumptions that in the vicinity of vacancy there is no other vacancy and only one impurity atom. In our samples both the configurations can exist but their influence on the annihilation characteristics does not seem to be dominant because of a low concentration of the impurity in the alloys investigated. Apart from that, the authors did not assume the direct proportionality between $E_{\mathrm{vi}}^{\mathrm{B}}$ and the number of v-i pairs but we think that there must be some relation between both the mentioned magnitudes. We also believe that the use of $E_{\mathrm{vi}}^{\mathrm{B}}$ is comfortable, because this energy reflects the valence fitting of alloy and matrix atoms, vacancy charge, dimension disproportion of atoms and other aspects deciding about attractiveness of v-i pairs in the alloy [13].

The second aspect of data analysis requiring a further discussion is based on the assumption that as a result of matrix relaxation in the vicinity of impurity atom the dimension of the surrounding defect changes too. The basis of this assumption lies on the intuitively well understandable deformation of the surrounding in the vicinity of a larger atom in the matrix. A simple geometrical analysis confirms that a vacancy existing in the vicinity of a bigger impurity atom must be bigger too.

The data on the atomic radii and the phase diagrams used for the calculation of the v-i binding energies are taken from [14-18] and references therein.

\section{Conclusions}

Through complementary information obtained from positron annihilation lifetime and Doppler broadening measurements as well as from the models of the thermodynamics of vacancies in dilute binary alloys we can state that for Ni alloys containing 1 at.\% of $\mathrm{Zn}, \mathrm{Ge}, \mathrm{Zr}, \mathrm{In}, \mathrm{Pb}, \mathrm{Ti}$ or $\mathrm{Sb}$

- there is a direct correlation between the first lifetime component and the product of vacancy-impurity binding energy and atomic radius of impurity atom,

- the Doppler broadening measurements confirm applicability of the newly constructed modernised version of Doppler apparatus in the field of demanding experiments with subtle differences between the measured spectra,

- still exist some questions and doubts concerning the problem of the interpretation of the lifetime spectra measured for the mentioned alloys, so the problem, in which way the positron annihilates in these systems, needs to be further studied,

- as a next step of our studies it will be looked at the pure alloying elements.

\section{References}

[1] G. Dlubek, R. Krause, O. Brümmer, Z. Michno, T. Górecki, J. Phys. F, Met. Phys. 17, 1333 (1987).

[2] E. Hashimoto, Y. Morita, T. Kino, J. Phys. F, Met. Phys. 14, 879 (1984).

[3] G. Dlubek, O. Brümmer, N. Meyendorf, Phys. Status Solidi A 53, K157 (1979). 
[4] M. Doyama, J. Nucl. Mater. 69/70, 350 (1978).

[5] A. Baranowski, M. Kostrzewa, M. Szuszkiewicz, Acta Phys. Pol. A 95, 439 (1999).

[6] P. Asoka-Kumar, M. Alatalo, V.J. Gosh, A.C. Kruseman, B. Nielsen, K.G. Lynn, Phys. Rev. Lett. 77, 2097 (1996).

[7] P. Kirkegaard, N.J. Pedersen, M. Eldrup, PATFIT-88: A Data Processing System for Positron Annihilation Spectra on Mainframe and Personal Computers, Tech. Rep. Ris $\varnothing-M-2740$, Ris $\varnothing$ National Laboratory, Roskilde (Denmark) 1989.

[8] M. Eldrup, B.N. Singh, J. Nucl. Mater. 251, 132 (1997).

[9] A. Vehanen, J. Yli-Kauppila, P. Hautojärvi, V.S. Mikhalenko, Phys. Status Solidi A 52, K73 (1979).

[10] A. Van Veen, A.C. Kruseman, H. Schut, P.E. Mijnarends, B.J. Kooi, J.Th. De Hosson, Mater. Sci. Forum 255-257, 76 (1997).

[11] W.M. Lomer, Vacancies and other Point Defects in Metals and Alloys, Institute of Metals, London 1958, p. 79.

[12] F.W. Schapink, Philos. Mag. 12, 1055 (1965); Acta Metall. 14, 1131 (1966).

[13] Y. Iijima, Phys. Status Solidi B 167, K13 (1991).

[14] P. Nash, Bulletin of Alloy Phase Diagrams 8/3 (1987).

[15] A. Dayer, P. Feschotte, J. Less-Common Met. 72, 51 (1980).

[16] W. Loskiewicz, M. Orman, Equilibrium Systems of Binary Alloys, PWN, Wrocław 1955 (in Polish).

[17] R.P. Elliot, Constitution of Binary Alloys. First Supplement, McGraw-Hill, New York 1965.

[18] G.B. Bokij, Krystalochemia, MGU, Moskva 1960. 